\title{
A Comparison of CP-OFDM with IOTA-OFDM Under Typical System Imperfections
}

\author{
Krishna P. Kongara \\ Peter J. Smith \\ Department of Electrical and Computer Engineering, \\ University of Canterbury, Christchurch, New Zealand
}

\author{
Stephen Mann \\ Tait Electronics Ltd \\ Christchurch, New Zealand
}

\begin{abstract}
In this paper we compare the performance of OFDM with a cyclic prefix (CP) and isotropic orthogonal transform algorithm (IOTA) OFDM without cyclic prefix under typical system imperfections such as channel estimation errors and frequency offset. The IOTA-OFDM system is based on offset quadrature amplitude modulation (OQAM) and promises an increased immunity to both inter-carrier interference (ICI) and inter-symbol interference (ISI). Moreover, IOTA-OFDM does not require a CP which makes it spectrally more efficient. Our simulation results show that compared to CP-OFDM, IOTAOFDM is spectrally efficient and more tolerant to interference and frequency offset. Imperfect channel estimation may reduce the gains of IOTA-OFDM but considerable throughput advantages are still obtained for a fixed BER.
\end{abstract}

\section{INTRODUCTION}

Orthogonal frequency division multiplexing systems in conjunction with offset quadrature amplitude modulation (OFDM/OQAM) have been proposed for fourth generation cellular systems, private mobile radio (PMR) and the IEEE 802.22 standard for wireless regional area networks (WRANs) [1], [2], [3]. As a result, OFDM systems based on OQAM have recently been a subject of research for next-generation mobile wireless systems [4], [5], [6], [7], [8]. Our work focuses on a comparison between conventional OFDM with a cylic prefix (CP) and isotropic orthogonal transform algorithm (IOTA) OFDM without a cyclic prefix.

In order to overcome the problems associated with conventional OFDM, the IOTA approach has been proposed [9], [10], [11]. OFDM/OQAM employing IOTA, as in [4], [5], [6], [7], is a unique approach. Since IOTA-OFDM does not use a guard interval it is spectrally more efficient. The IOTA-OFDM system employs well localized orthogonal pulses in both time and frequency, and hence provides better immunity against both ISI and ICI. The effect of adjacent channel interference (ACI) on IOTA-OFDM is not so severe, as the spectrum of an IOTA function fades much faster, compared to the spectrum of a rectangular pulse. As proposed in [4], [6], [12], [13], the efficient use of fast Fourier transform (FFT) algorithms and polyphase filtering for IOTA can be shown to simplify the implementation complexity of the IOTA-OFDM system.

Many papers [4], [5], [6], [7], [14] have demonstrated promising advantages of OFDM/OQAM over CP-OFDM and this technology has already been introduced in many standards [1], [2], [3]. Due to the intrinsic ISI of the IOTA function, the conventional pilot aided channel estimation (PACE) schemes used in CP-OFDM are not directly applicable to IOTA-OFDM. In [15], a preamble-based channel estimation procedure for OFDM/OQAM was proposed. However, performance comparisons between CP-OFDM and IOTA-OFDM are still not comprehensive. In this paper we consider the effects of channel estimation error and frequency offset in a frequency selective channel with Doppler effects and additive noise. System comparisons are made on the basis of bit error rate (BER) and we are able to assess the joint and individual effects of the system imperfections. Our simulation results show that compared to CP-OFDM, IOTA-OFDM is spectrally efficient and more tolerant to interference and frequency offset. Imperfect channel estimation may reduce the gains of IOTA-OFDM but considerable throughput advantages are still obtained for a fixed BER.

The rest of the paper is organized as follows. In Sec. II we outline key assumptions and notation used and present the system model for CP-OFDM and IOTA-OFDM. Sec. III introduces the channel model. Simulation results and conclusions follow in Sec. V and Sec. VI.

\section{SySTEM MODEL}

We assume a familiarity with the operation of classical CPOFDM systems. IOTA-OFDM/OQAM is less widely reported so some details of the transmitter and receiver structures will be discussed in this section.

\section{A. IOTA-OFDM Transmitter Model}

The key technique of OFDM/OQAM is the transmission of the real and imaginary parts of complex QAM symbols by offsetting them in time. [6], [9]. In its general form, the OFDM/OQAM baseband equivalent signal can be formed by summing N OQAM modulations. The transmitted signal in the continuous time domain can be written as

$$
s(t)=\sum_{m=0}^{N-1} \sum_{n=-\infty}^{\infty} C_{m, n} e^{j(m+n) \pi / 2} e^{j 2 \pi m v_{o} t} \zeta_{m, n}\left(t-n T_{o}\right)
$$

where $T_{o}$ is the time interval between two successive symbols on a given subchannel and $v_{o}$ is the frequency spacing between two adjacent subchannels. $C_{m, n}$ is the symbol transmitted on the $m^{\text {th }}$ subchannel during the $n^{t h}$ symbol period and $N$ is the number of subchannels. The IOTA function, $\zeta_{m, n}$, is obtained 
by repeated orthogonalisation of the Gaussian function $g(t)=$ $2^{\frac{1}{4}} e^{-\pi t^{2} / 2 T_{o}^{2}}$ in both time and frequency using the methods presented in [6], [11]. Following [11], in the Appendix we provide a method to derive the necessary coefficients for computing the IOTA function. The IOTA function allows a subchannel spacing of half the symbol rate $\left(v_{o}=1 / 2 T_{o}\right)$ provided that the symbols from in-phase and quadrature channels are transmitted in an alternating fashion. This type of modulation is commonly known as IOTA-OFDM/OQAM or simply OFDM/OQAM. Fig. 1 shows the block diagram of the transmitter. The input data is mapped to a signal point of LQAM, serial to parallel converted and the OQAM signal is generated by alternately delaying the real and imaginary parts by $T_{o}$. The output of the IFFT unit is passed through an IOTA pulse shaping filter and the resulting signal $s(t)$ is transmitted.

The implementation complexity of IOTA-OFDM is slightly higher than CP-OFDM, since the prototype function $\zeta_{m, n}(t)$ is wider than $T_{o}$. However, from Table I, the coefficients of the filter fall off rapidly and hence the filter can be safely truncated to 14-taps. In addition, the complexity can be further reduced by using polyphase implementation [16].

\section{B. IOTA-OFDM Receiver Model}

At the receiver side, the received signal $r(t)$ can be written as

$r(t)=h(t) * s(t)+n(t)=\sum_{m=0}^{N-1} \sum_{n=-\infty}^{\infty} h_{m, n} s\left(t-n T_{o}\right)+n(t)$

where $h(t)$ is the wireless channel impulse response and $h_{m, n}$ represents the channel realization on the $m^{t h}$ subchannel and $n^{t h}$ symbol period. The noise term is denoted by $n(t)$ and is modeled as AWGN according to $\mathcal{C N}\left(0, \sigma^{2}\right)$.

The receiver shown in Fig. 1 uses the conjugate of the structure used at the transmitter. Passing $r(t)$ through $N$ parallel correlator demodulators with the conjugate structure, the output of the $m^{\text {th }}$ branch during the time interval $(n-$ 1) $T_{o} \leq t<n T_{o}$ is

$\widetilde{C}_{m, n}=\sum_{m=0}^{N-1} \sum_{n=-\infty}^{\infty} r(t) e^{-j(m+n) \pi / 2} e^{-j 2 \pi m v_{o} t} \zeta_{m, n}^{*}\left(t-n T_{o}\right)$

Using the orthogonality conditions of the IOTA function [11], [6], [7], this output can be expressed as

$$
\widetilde{C}_{m, n}=H_{m, n} C_{m, n}+N_{m}
$$

where $H_{m, n}$ represents the channel gain (in the frequency domain) associated with $m^{\text {th }}$ subchannel and $n^{\text {th }}$ symbol period after the IOTA filtering and FFT operation. $N_{m}$ is the noise associated with $m^{\text {th }}$ subchannel after the receiver processing described above. The symbols are detected after a simple zero forcing (ZF) stage using the estimated channel transfer gain $\widehat{H}_{m, n}$,

$$
\widehat{C}_{m, n}=\widetilde{C}_{m, n} \frac{\widehat{H}_{m, n}^{*}}{\left|\widehat{H}_{m, n}\right|^{2}} .
$$

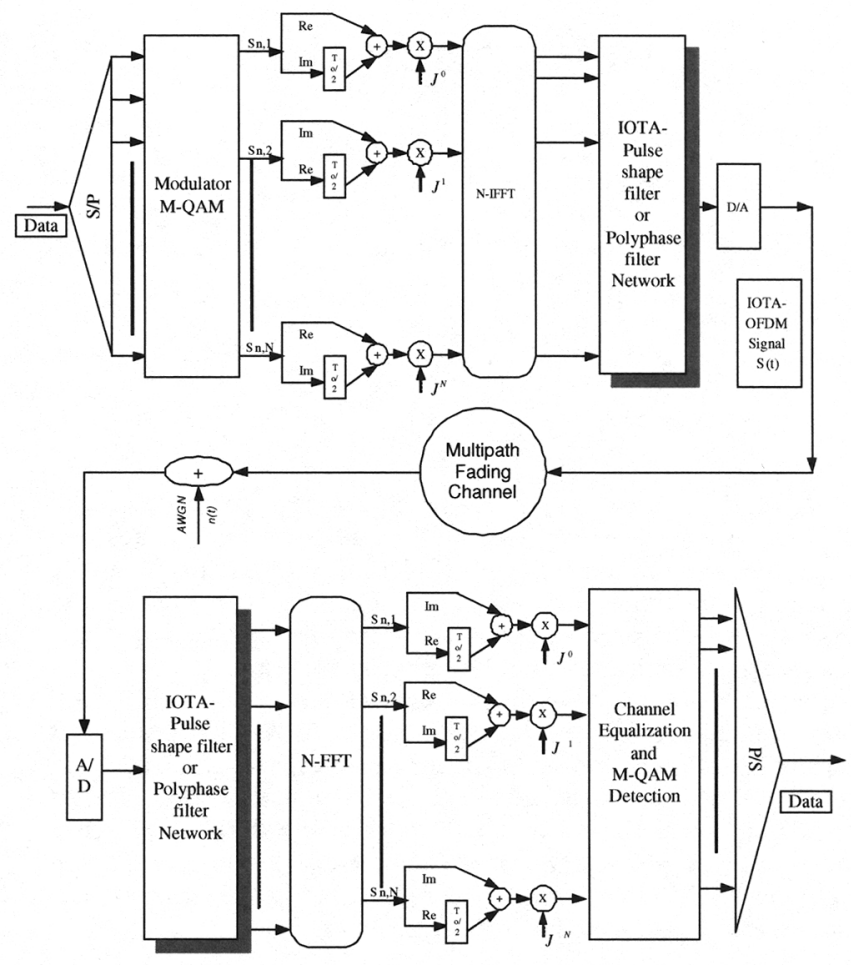

Fig. 1. IOTA-OFDM/OQAM Baseband Transceiver Model

Unlike CP-OFDM, in IOTA-OFDM a simple ZF doesn't cancel the residual or intrinsic interference due to the time dispersive nature of the channel. To avoid such interference a preamble-based channel estimation for OFDM/OQAM was proposed in [15]. However, here we are not interested in using a specific type of channel estimation/equalization. In this paper, we are interested in the performance evaluation of CPOFDM and IOTA-OFDM with comparable channel estimation errors.

\section{Frequency Selective Channel Model}

We assume a familiarity with frequency selective Rayleigh fading channels and use the well-known Jakes' model [17]. We make the general assumption of a frequency selective Rayleigh fading channel that is wide sense stationary with uncorrelated, isotropic scattering. Furthermore, we presume that the delay autocorrelation function may be described as an exponential delay power profile with rms delay $\tau_{d}$. The subchannel gains of the $k_{1}$-th and $k_{2}$-th subcarriers can be written as

$$
H_{k_{1}}=X_{k_{1}}+j Y_{k_{1}} \text { and } H_{k_{2}}=X_{k_{2}}+j Y_{k_{2}}
$$

where $X_{k_{1}}, Y_{k_{1}}, X_{k_{2}}$ and $Y_{k_{2}}$ are identically distributed zero mean Gaussian random variables. For ease of notation, in this Section we omit the time subscript for the channel gains in the frequency domain. Hence, $H_{k, n}$ is denoted by $H_{k}$. Without loss of generality we may set $\mathrm{E}\left[X_{k}^{2}\right]=\mathrm{E}\left[Y_{k}^{2}\right]=\frac{1}{2}$, for all $k$. Following [17], we may then write the cross-correlation 
properties

$$
\begin{aligned}
\mathrm{E}\left[X_{k_{1}} X_{k_{2}}\right] & =\mathrm{E}\left[Y_{k_{1}} Y_{k_{2}}\right]=\frac{1}{2} \frac{1}{1+\left(2 \pi \tau_{r m s} v_{o} \Delta k\right)^{2}} \\
\mathrm{E}\left[X_{k_{1}} Y_{k_{1}}\right] & =\mathrm{E}\left[X_{k_{2}} Y_{k_{2}}\right]=0 \\
\mathrm{E}\left[X_{k_{1}} Y_{k_{2}}\right] & =-\mathrm{E}\left[X_{k_{2}} Y_{k_{1}}\right]=-\left(2 \pi v_{o} \Delta k \tau_{r m s}\right) \mathrm{E}\left[X_{k_{1}} X_{k_{2}}\right.
\end{aligned}
$$

where $\Delta k=\left|k_{1}-k_{2}\right|$. With these definitions we obtain the correlation function

$$
\rho_{f}\left(\Delta k v_{o}\right)=\mathrm{E}\left[H_{k_{1}} H_{k_{2}}^{*}\right]=\frac{1+j 2 \pi \tau_{r m s} v_{o} \Delta k}{1+\left(2 \pi \tau_{r m s} v_{o} \Delta k\right)^{2}} .
$$

Note that from (6), the marginal distribution of each channel gain $\left|H_{k}\right|^{2}$ follows an exponential distribution with $\mathrm{E}\left[\left|H_{k}\right|^{2}\right]=1, \operatorname{var}\left|H_{k}\right|^{2}=1$ and

$$
\operatorname{corr}\left(\left|H_{k}\right|^{2},\left|H_{k+\Delta k}\right|^{2}\right)=\frac{1}{1+\left(2 \pi v_{o} \Delta k \tau_{r m s}\right)^{2}}
$$

where $\operatorname{corr}(\cdot, \cdot)$ represents the correlation coefficient.

\section{SYSTEM IMPERFECTIONS}

\section{A. Channel Estimation Errors}

Here we consider that the estimated channel is correlated with the true channel via the correlation, $\rho_{e}=$ $\mathrm{E}\left[\widehat{H}_{m, n}, H_{m, n}\right]$. Hence, the estimated channel can be obtained by using [18]

$$
\widehat{H}_{m, n}=\rho_{e} H_{m, n}+\sqrt{1-\rho_{e}^{2}} E_{m, n}
$$

where $E_{m, n}$ is the Gaussian error signal and is assumed independent of the true channel realization. To keep the same behavior across frequency for the $\widehat{H}_{m, n}$ process as for the $H_{m, n}$ process we assume that the $E_{m, n}$ has the same statistical properties as $H_{m, n}$. Hence $E_{m, n}$ satisfies (6) - (9).

A statistical model for imperfect CSI is used as it allows a comparison of IOTA-OFDM and CP-OFDM under exactly the same conditions. Hence, any differences in performance are due to the fundamental dependence of the schemes on CSI, rather than the particular channel estimation procedures used. To calibrate the statistical model a PACE procedure [19] was also simulated for CP-OFDM. The results are similar to the statistical model in (10) with correlation $\rho_{e}=0.999$. Since a practical estimation procedure yields results which closely match $\rho_{e}=0.999$, we restrict our simulations to the region $\rho_{e} \geq 0.99$.

\section{B. Frequency Offset}

Carrier frequency offset is introduced during transmission because of channel distortions, such as Doppler frequency shift, or at the receiver due to crystal oscillator inaccuracy. This error will normally be compensated in the receiver prior to demodulation. Since the compensation will not be perfect, a certain carrier frequency offset $\delta f$ will always be present. In this paper, we present performance comparisons between CP-OFDM and IOTA-OFDM under such scenarios.

\section{Simulation Results}

The simulations were carried out for a 64 subcarrier system with a separation of $v_{o}=0.3125 \mathrm{MHz}$, thus occupying a bandwidth of $20 \mathrm{MHz}$. Also, a system carrier frequency of $5.725 \mathrm{GHz}$ (HyperLan 2 standard) is chosen. Figures $2-5$ show the simulated BER versus mean SNR $\left(E_{b} / N_{o}\right)$ for a Rayleigh fading channel with an exponentially decaying power delay profile. For CP-OFDM a CP of length $G$ equal to 16samples was used and for IOTA-OFDM a pulse shaping filter of length 14-taps with the coefficients given in Table I was used. Figure 2 shows the BER performance for both systems with perfect CSI at the receiver. IOTA-OFDM performs better than CP-OFDM as the cyclic prefix alone cannot combat the interference due to multipath fading channel. For example, at a BER of $10^{-3}$ IOTA-OFDM is approximately $4 d B$ better off than CP-OFDM. From Figure 3, in the presence of imperfect channel state information (CSI), this gain may be slightly reduced. Furthermore, both techniques show a great sensitivity to the level of channel estimation error. Overall, the effect of imperfect CSI is to push the BER curves up towards a floor. This has the effect of narrowing the gap between the IOTA-OFDM and CP-OFDM results. Note that a considerable throughput increase is achieved by not using a CP. Specifically, the throughput improvement is $\frac{100 N}{G} \%$, which gives $25 \%$ for the particular system under consideration.

Figure 4 displays the effect of frequency offset on CPOFDM and the IOTA-OFDM. Simulations were carried out for three different values of $\delta f$. Here, we can see that in both systems, the distortion introduced due to frequency offset is severely degrading the BER performance. Due to the use of well localized pulses in the frequency domain, the IOTAOFDM is less sensitive to frequency offset errors. However, for higher $\delta f$, in both systems, there is an irreducible error floor in BER. This suggests that accurate estimation of frequency offset plays a crucial role in achieving good performance. Figure 5 demonstrates the effect of varying delay spread on the system performance in the presence of both frequency offset and imperfect channel estimation over a fading noisy channel. Simulations are performed for $\tau_{r m s}$ values of $50 \mathrm{~ns}$ and 80ns. Here, we can see the inherent robustness of IOTAOFDM to the time dispersion in the propagation environment. The results in Fig. 5 show the joint effect of all the channel effects and system impairments considered. The improvements of IOTA-OFDM in improved immunity to interference and reduced sensitivity to frequency offset are partially reduced by imperfect CSI. However, BER advantages of several $\mathrm{dB}$ are still realized with a throughput advantage of $25 \%$. If BER results were fixed for the two systems then the throughput advantages of IOTA-OFDM would be considerable ( $₫ 25 \%)$.

\section{CONCLUDING REMARKS}

In this paper, we have considered some fundamental issues concerning the BER performance of CP-OFDM and IOTAOFDM under typical system imperfections. Simulation results show that IOTA-OFDM performs better than CP-OFDM. In addition to a fundamental throughput advantage due to the 


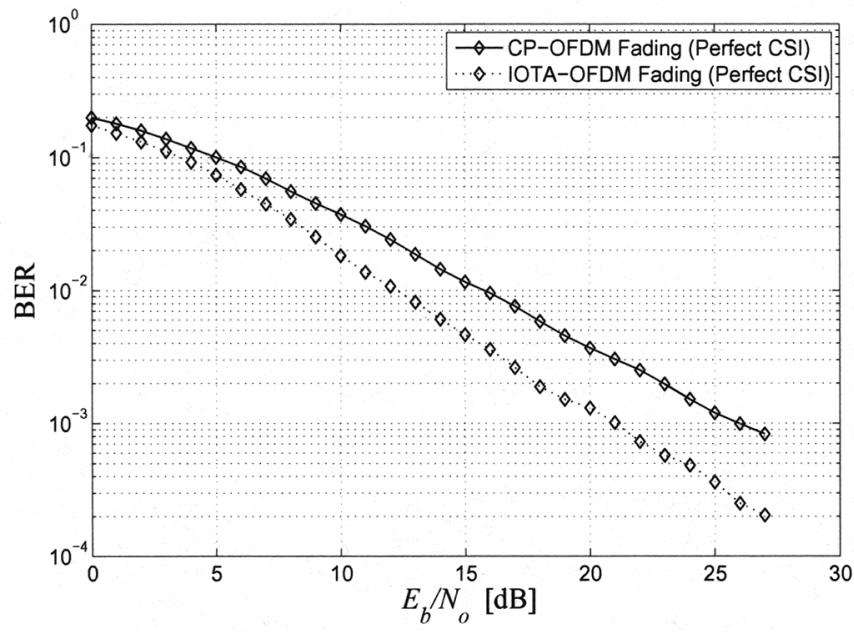

Fig. 2. Uncoded BER comparison between CP-OFDM and IOTA-OFDM with perfect CSI at the receiver $\left(\tau_{r m s}=50 n s\right)$.

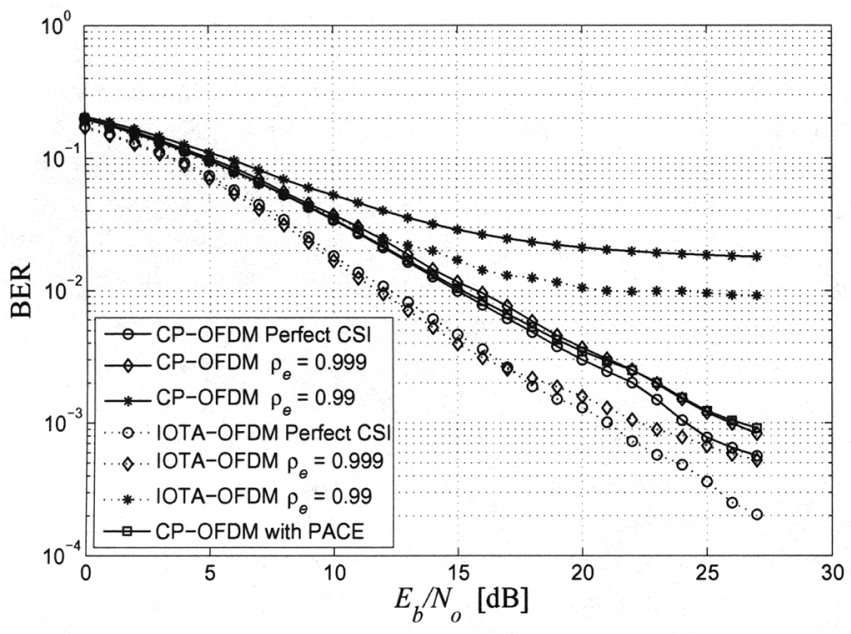

Fig. 3. Uncoded BER comparison between CP-OFDM and IOTA-OFDM over a Rayleigh fading channel with channel estimation errors at the receiver $\left(\tau_{r m s}=50 n s\right)$.

lack of a CP, IOTA-OFDM also offers improved immunity to interference and reduced sensitivity to frequency offset. This creates substantial throughput improvements for comparable BER values.

\section{APPENDIX}

Using the method presented in [9], [11] the coefficients for the orthogonal pulse $g_{o}(t)$ can be obtained. The Gaussian pulse, $g(t)$, is orthogonalized using,

$$
g_{o}(t)=O_{T_{o}} F^{-1} O_{v_{o}} F g(t) .
$$

In (11), $O_{a}$ is an orthogonalization operator with $a$ equal to $T_{o}$ or $v_{o}$ so that it orthogonalizes the function along the time or frequency axis. Also in (11), $F$ is the Fourier transform operator. Specifically, the orthogonalization operator

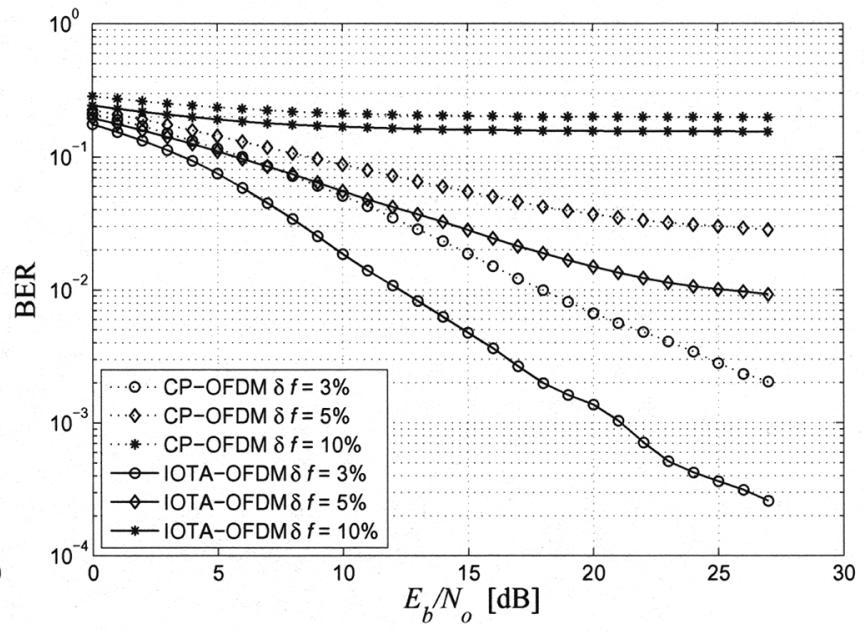

Fig. 4. Uncoded BER comparison between CP-OFDM and IOTA-OFDM with frequency offset errors at the receiver and assuming perfect CSI $\left(\tau_{r m s}\right.$ $=50 n s)$.

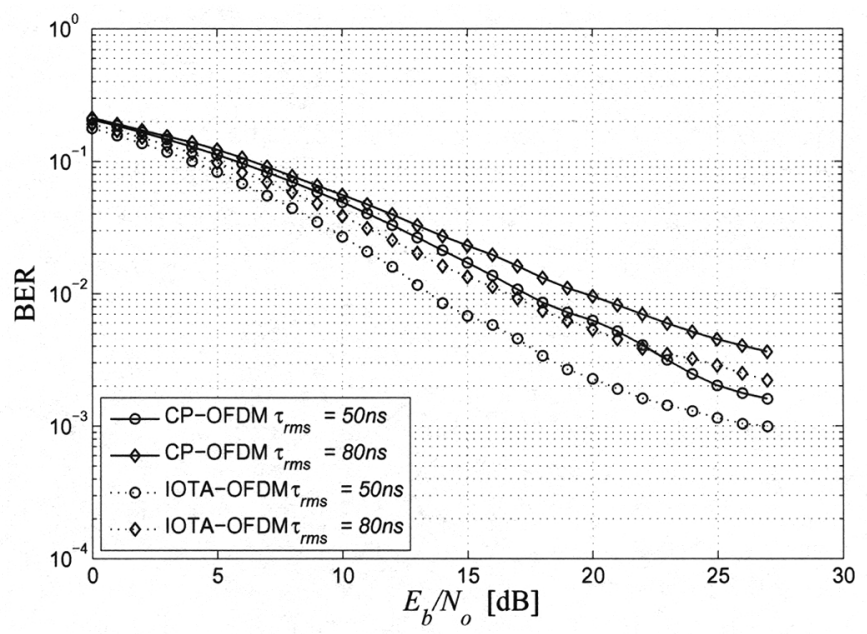

Fig. 5. Uncoded BER comparison between CP-OFDM and IOTA-OFDM with frequency offset $(\delta f=3 \%)$ and with channel estimation errors $\left(\rho_{e}=\right.$ 0.999 ) at the receiver.

$O_{a}$ transforms a function $x$ into $y$ according to

$$
y(u)=\frac{x(u)}{\sqrt{a \sum_{m=-\infty}^{+\infty}\|x(u-m a)\|^{2}}} .
$$

The first eight coefficients required by the pulse shaping filter are computed from (11) and (12) and are given in Table I. As can be observed, the coefficients die away extremely rapidly. In our implementation, only the first seven coefficients are used. Note that the coefficients of the pulse shaping filter obtained using (11) and (12) ensure the orthogonality between two adjacent time or frequency symbols. Hence, at the receiver, we can recover the transmitted symbols. 
TABLE I

COEFFICIENTS OF THE PULSE SHAPE FILTER OBTAINED USING IOTA

\begin{tabular}{ll}
\hline Coefficient Index & Value \\
\hline$g_{o}(0)$ & 1.00000 \\
$g_{o}(1)=g_{o}(-1)$ & $-2.155710^{-2}$ \\
$g_{o}(2)=g_{o}(-2)$ & $7.100710^{-4}$ \\
$g_{o}(3)=g_{o}(-3)$ & $-2.461710^{-5}$ \\
$g_{o}(4)=g_{o}(-4)$ & $9.598910^{-7}$ \\
$g_{o}(5)=g_{o}(-5)$ & $-3.800610^{-8}$ \\
$g_{o}(6)=g_{o}(-6)$ & $1.511210^{-9}$ \\
$g_{o}(7)=g_{o}(-7)$ & $-5.795910^{-11}$ \\
$g_{o}(8)=g_{o}(-8)$ & $-8.959110^{-13}$ \\
\hline
\end{tabular}

\section{REFERENCES}

[1] "Wideband air interface isotropic orthogonal transform algorithm (IOTA) - public safety wideband data standards project - digital radio technical standards TR-8.5 subcommittee," TIA902.BBAB (Physical Layer Specification, March 2003).

[2] "Wireless regional area networks (WRAN) - physical layer (PHY) specifications," IEEE802.22, 2004.

[3] "Radio broadcast systems; digital audio broadcasting (DAB) to mobile, portable and fixed receivers," ETS300401, 1994.

[4] P. Siohan, C. Siclet, and N. Lacaille, "Analysis and design of OFDM/OQAM systems based on filterbank theory," IEEE Trans. Acoust., Speech, Signal Processing, vol. 50, no. 5, pp. $1170-1183$, May 2002.

[5] D. Lacroix, N. Goudard, and M. Alard, "OFDM with guard interval versus OFDM/offsetQAM for high data rate UMTS downlink transmission," in Proc. IEEE Vehicular Technology Conf., vol. 4, Atlantic City, NJ, 7-11 Oct 2001, pp. 2682 - 2686.

[6] H. Bolcskei, P. Duhamel, and R. Hleiss, "Design of pulse shaping OFDM/OQAM systems for high data-rate transmission over wireless channels," in Proc. IEEE Int'l. Conf. on Communications, vol. 1, Vancouver B.C., Canada, 6-10 June 1999, pp. 559 - 564.

[7] P. Siohan and R. Christian, "Derivation of extended Gaussian functions based on the Zak transform," IEEE Trans. Signal Processing, vol. 11, no. 3 , pp. $401-403,2004$.

[8] A. Valin and N. Holte, "Optimal finite duration pulse for OFDM," IEEE Trans. Commun., vol. 44, no. 1, pp. $10-14$, Jan. 1996.

[9] P. Siohan and C. Roche, "Cosine modulated filterbanks based on extended gaussian functions," IEEE Trans. Signal Processing, vol. 48, pp. 3052-3061, 2000.

[10] W. Y. Zou and Y. Wu, "COFDM: An overview," IEEE Trans. Broadcast., vol. 40, no. 1, pp. 1-8, 1995.

[11] B. L. Floch and M. Alard, "Coded orthogonal frequency division multiplex," Proc. IEEE, vol. 83, no. 6, pp. 982-996, 1995.

[12] H. Bolcskei, P. Duhamel, and R. Hleiss, "Design of pulse shaping OFDM/OQAM systems for wireless communications with high spectral effeciency," IEEE Trans. Signal Processing, vol. 11, no. 3, pp. 401 403, 1998.

[13] M. Muck and J. Javaudin, "Advanced OFDM modulators considered in the IST-WINNER framework for future wireless systems," Mobile Summit 05, Dresden, Germany, June 2005.

[14] J. Du and S. Signell, "Classic OFDM systems and pulse shaping OFDM/OQAM systems," Electronic, Computer, and Software Systems Information and Communication Technology KTH - Royal Institute of Technology SE-100 44 Stockholm, Sweden, February 2007.

[15] C. Lele, P. Siohan, and R. Legouable, " $2 \mathrm{db}$ better than CP-OFDM with OFDM/OQAM for preamble-based channel estimation," in Proc. IEEE Int'l. Conf. on Communications, Beijing, China, 19-23 May 2008, pp. $1302-1306$.

[16] P. P. Vaidyanathan, Ed., Multirate systems and filter banks. Englewood Cliffs, NJ, USA: Prentice Hall, 1992.

[17] W. C. Jakes, Ed., Microwave Mobile Communications. New York, NY, USA: IEEE Press, 1994.

[18] P. J. Smith, T. W. King, L. M. Garth, and M. Dohler, "An analysis of low complexity algorithms for MIMO antenna selection," in Proc. IEEE Int'l. Conf. on Communications, vol. 3, Istanbul, Turkey, 11-15 June 2006, pp. 1380 - 1385.
[19] L. J. Cimini, "Analysis and simulation of a digital mobile channel using orthogonal frequency division multiplexing," IEEE Trans. Commun., vol. 33, pp. 665-675, July 1985. 